\title{
Intelligent Ultrasonic Energy: New Adaptive Tissue Technology in Harmonic Shears
}

\author{
David Singleton ${ }^{1, \text { * }, \text { Natalia Juncosa-Melvin }}{ }^{2}$, Patrick Scoggins ${ }^{3}$, Geisa Paulin-Curlee ${ }^{2}$, \\ John Cummings ${ }^{2}$, Crystal Ricketts ${ }^{1}$ \\ ${ }^{1}$ Department of Medical Affairs, Ethicon, Inc., Blue Ash, Ohio, USA \\ ${ }^{2}$ Department of Preclinical Research, Ethicon, Inc., Blue Ash, Ohio, USA \\ ${ }^{3}$ Department of Research and Development, Ethicon, Inc., Blue Ash, Ohio, USA
}

Email address:

dsingl12@its.jnj.com (D. Singleton)

${ }^{*}$ Corresponding author

\section{To cite this article:}

David Singleton, Natalia Juncosa-Melvin, Patrick Scoggins, Geisa Paulin-Curlee, John Cummings, Crystal Ricketts. Intelligent Ultrasonic Energy: New Adaptive Tissue Technology in Harmonic Shears. Journal of Surgery. Vol. 8, No. 6, 2020, pp. 178-183. doi: $10.11648 /$ j.js.20200806.12

Received: October 6, 2020; Accepted: October 23, 2020; Published: November 4, 2020

\begin{abstract}
Objective: Development of ultrasonic technology in surgical devices includes mechanisms of monitoring and adjusting energy delivery to target tissues for the purpose of limiting thermal spread. The objective of the current study was to evaluate performance of a new Adaptive Tissue Technology algorithm, designed to enhance thermal management in the HARMONIC 1100 Shears. Methods: The HARMONIC 1100 Shears were evaluated with bench-top and in vivo preclinical (porcine) analyses for tissue thermal spread, blade heat, transection speed, hemostasis and vessel sealing performance. Testing was performed in parallel with the current production HARMONIC HD 1000i Shears to confirm non-inferiority of the new Adaptive Tissue Technology algorithm. Results: Bench top analysis revealed significantly lower average maximum blade temperatures for HARMONIC 1100 compared to HARMONIC HD 1000i as well as HARMONIC ACE 7 Shears with Advanced Hemostasis. Thermal spread, transection speeds, and burst pressure tests of excised porcine carotid arteries did not show a statistical difference between HARMONIC 1100 and HD 1000iShears. In vivo analysis of hemostasis following sealing/transection of various blood vessels in acute porcine testing demonstrated similar efficacy for HARMONIC 1100 and HD 1000i Shears. Likewise, tissue lateral thermal damage showed no statistical difference between the new and previous generation Harmonic device in the porcine model. Conclusion: The new Adaptive Tissue Technology in HARMONIC 1100 Shears allows for reduced maximum blade temperatures while providing effective hemostasis, sealing strength, transection speed, and limited thermal damage.
\end{abstract}

Keywords: Ultrasonic, Harmonic, Laparoscopy, Tissue Sealing, Hemostasis

\section{Introduction}

The increasing complexity of minimally invasive and open surgical procedures necessitates continuous development of new devices and surgical techniques. This includes improvement of electrosurgical device technology to provide reliable and precise dissection of tissue in combination with adequate hemostasis. Ultrasonic energy devices represent a technology that has undergone substantial development and refinement over the past few decades and are now used in many surgical specialties for tissue cutting and coagulation.
Reported advantages of ultrasonic energy compared to conventional electrosurgery instruments include improved hemostasis, less thermal damage, combined vessel sealing / tissue dissection capability, and less surgical smoke production [1-4]. Hemostasis is achieved by coaptation and sealing of vessels with a denatured protein coagulum produced by transduction of mechanical energy to the tissue. In addition, modern ultrasonic devices such as Harmonic shears (Ethicon, Inc.) may provide a smaller more precise footprint, and can seal vessels up to $7 \mathrm{~mm}$ in diameter as opposed to the traditional $5 \mathrm{~mm}$ limit for vessels sealed with 
earlier ultrasonic devices. This feature can mitigate the need for separate instruments to perform hemostatic dissection and transection of larger vessels. Clinical meta-analyses have demonstrated that Harmonic devices are associated with significant reductions in operating time [5-8], blood loss [5, 6, 8,9], post-operative pain [8], complications [9], length of hospital stay $[5,9]$, and costs $[10,11]$.

The current generation of Harmonic shears (HD 1000i, $\mathrm{ACE}+7$, and $\mathrm{ACE}+$ ), are multifunctional devices that can be used for dissection, coaptation, coagulation and transection during laparoscopic or open surgical procedures. Recently, a new Adaptive Tissue Technology system was developed to deliver a more precise amount of thermal energy during tissue transection and vessel sealing. This new Adaptive Tissue Technology algorithm actively limits the maximum temperature of the ultrasonic blade to lower levels, which is designed to provide enhanced protection of tissues and surrounding vital structures and still deliver effective cutting and sealing. This feature may also improve efficiency by allowing surgeons to fully focus on the procedure, while maintaining confidence that the device provides effective thermal management. The aim of the current study was to evaluate HARMONIC 1100 Shears (Figure 1), in comparison to the previous version, HARMONIC HD 1000i Shears for vessel sealing performance, lateral thermal spread, and temperature management using bench-top and preclinical models.

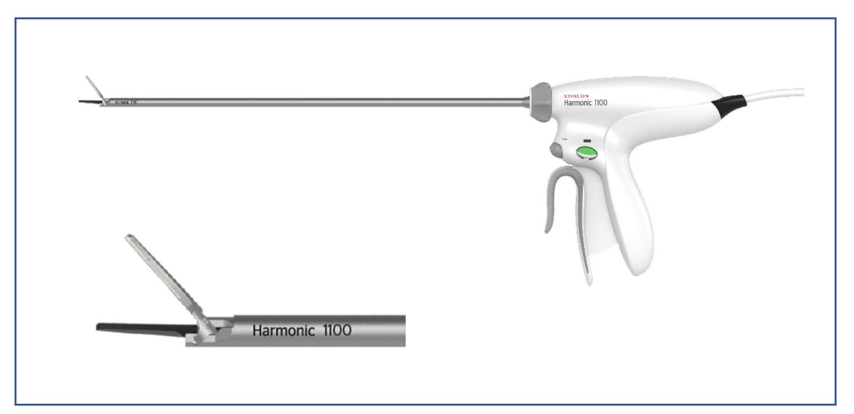

Figure 1. HARMONIC 1100 Shears with next generation Adaptive Tissue Technology.

\section{Methods}

\subsection{Study Device}

Devices tested in this study included the HARMONIC 1100 Shears in 20 and $36 \mathrm{~cm}$ length (HAR1120 and HAR1136) powered by the GEN11 generator (Ethicon, Inc., Cincinnati $\mathrm{OH})$. The devices were evaluated in bench-top and preclinical (porcine) analyses in parallel with the predicate device, for confirmation of non-inferiority of the updated shears. The analyses were done as verification of performance of the new Adaptive Tissue Technology algorithm. The HARMONIC 1100 Shears are mechanically identical to the previous generation HD 1000i Shears, with the only difference being the new version Adaptive Tissue Technology with an enhanced thermal control.

\subsection{Benchtop Studies}

\subsubsection{Lateral Thermal Spread}

To assess the thermally affected zone for HARMONIC 1100 and HD 1000i Shears, ex vivo porcine carotid arteries of 3-5 mm external diameter were transected with the devices' energy button set to power level 5, with use of the GEN11 generator. An infrared (IR) thermal camera was placed in position to capture images of the end effector and artery during transection, specifically at the moment just prior to separation of the vessel. Thermally affected zone data (edge to edge linear distance along the artery on either side of the device jaws) were analyzed with ThermaCAM Researcher Professional software.

\subsubsection{Maximum Transient Blade Temperature}

Devices were activated with 15 consecutive tip bites (marching transections) across controlled test media while maximum transient temperatures were determined for each of the activations using the IR thermal camera and analysis with ThermaCAM software. All devices made 3 sets of 15 marching cuts, with a 5-minute cool-down between sets. The overall mean transient maximum temperatures associated with each device were determined from the 3 trials. Tests were performed for the HARMONIC 1100, HD 1000i, and $\mathrm{ACE}+7$ shears with energy power levels set at 5 .

\subsubsection{Marching Transection Speed}

The average duration for transection across excised porcine jejunum was determined for the Harmonic devices using the energy button set at power level 5. The tip bite transection speed as well as full bite transection speed were tested. For the tip bite analysis, the distal $5 \mathrm{~mm}$ of the device jaws were marked to enable utilization of only this portion of the end effector. Each instrument was timed for completion of nine cuts across the porcine jejunum for the tip bite speed, and 8 cuts for the full bite analysis.

\subsubsection{Burst Pressures}

Excised porcine carotid arteries (3-5 mm diameter) were sealed and transected with the devices' energy button set at power level 5. Transections were performed with arteries placed in the proximal, middle, or distal portion of the device jaws. The left and right halves of sealed vessels were subjected to testing with a burst pressure system by infusion of physiologic saline into the transected vessel halves. The intra-luminal pressure was recorded until maximum burst pressure was reached at seal failure. The left and right bursting pressures were pooled and averaged according to jaw location of the transections.

\subsection{Preclinical Evaluations}

\subsubsection{Porcine Hemostasis Model}

A total of 20 female pigs were used for acute evaluation of hemostasis. All in vivo procedures were reviewed, and animals approved for use in the study by the Institutional Animal Care and Use Committee. The 2 lengths of HARMONIC 1100 Shears $(20$ and $36 \mathrm{~cm}$ ) were tested on 5 
pigs each, and likewise the 2 lengths of HD 1000i Shears (20 and $36 \mathrm{~cm}$ ) were tested on 5 pigs each. Each pig had 2 rounds of vessel sealing/transection followed by a simulated hypertensive crisis (blood pressure challenge). The Energy Activation and Advanced Hemostasis modes were alternated between rounds. For the Advanced Hemostasis mode, 17 vessels per animal were targeted up to and including $7 \mathrm{~mm}$ in diameter, including gastroepiploic pedicle, short gastric pedicles, splenic artery and vein, renal artery and vein and carotid artery. For the Energy Activation mode, 19 vessels per animal were targeted up to and including $5 \mathrm{~mm}$ in diameter, including gastroepiploic artery and veins, short gastric pedicles, splenic artery and vein, uterine artery, ovarian pedicle and carotid artery. The same vessels were targeted for the HD1000i devices. All vessel seals were evaluated for hemostasis at initial application (first pass hemostasis) and after a simulated hypertensive crisis, wherein the animal's blood pressure was increased to at least $200 \mathrm{~mm} \mathrm{Hg}$ for 10 minutes using phenylephrine as a vasopressor agent. In the thoracic cavity, the HARMONIC 1100 and HD $1000 \mathrm{i} 20 \mathrm{~cm}$ devices were used to seal and transect pulmonary arteries and veins in 4 female pigs. Two pigs were used for HARMONIC 1100 Shears hemostasis evaluation and another two pigs for HD 1000i hemostasis evaluation. A total of 30 vessels were targeted per animal.

\subsubsection{Surgical Procedures}

After a standard procedure of induction of anesthesia with Telazol/Xylazine, and maintenance with isoflurane, with the pig in dorsal recumbency, an arterial catheter was placed to directly monitor blood pressure and a phenylephrine drip was administered if systolic blood pressure fell below $80 \mathrm{~mm} \mathrm{Hg}$. Splenectomy, nephrectomy, and hysterectomy were performed through a midline laparotomy and carotid artery transection via a midline neck incision. During the splenectomy, the gastroepiploic pedicle or artery and veins, short gastric pedicles, and splenic artery and vein were isolated, the diameters were measured, and then the vessels were sealed and transected. During the nephrectomy, the renal arteries and veins were isolated, the diameters were measured, and the vessels were sealed and transected. For the hysterectomy, the ovarian pedicle and uterine artery were sealed and transected. In the thoracic study, a combination of pulmonary arteries, pulmonary veins, and azygous veins were sealed and transected using HARMONIC 1100 and HD $1000 \mathrm{i}$ Shears. The vessels were accessed through a thoracotomy on the left side.

\subsubsection{Assessment of Thermal Damage and Tissue Dissection Quality}

The extent of lateral thermal damage was assessed via histological evaluation of adventitial collagen denaturation of the carotid artery seal using hematoxylin and eosin staining. To evaluate tissue dissection capability, HARMONIC 1100 was compared to the HARMONIC HD 1000i in non-energy, mechanical dissection. Each device was used to isolate the iliac artery from the iliac vein and surrounding tissue, and to mobilize a lymph node in the base of the small bowel mesentery. Dissection capability was subjectively assessed by the surgeon.

\subsection{Statistical Methods}

All continuous variable data sets in benchtop analyses were assessed for normal distribution, and paired t-tests were used for comparisons of normally distributed data. An alpha level of significance was taken as 0.05 . For preclinical in vivo analysis, a non-inferiority test with a $10 \%$ non-inferiority margin was used for statistical analysis of first pass hemostasis and for the hemostasis after blood pressure challenge. To evaluate the non-inferiority hypothesis at the one-sided 0.05 level of significance, a two-sided $90 \%$ confidence interval for the difference in proportions (pass ${ }_{\text {Test }}$

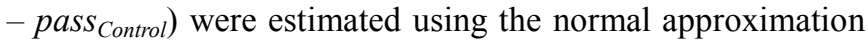
to the binomial distribution. If the lower bound of the confidence interval was strictly greater than -0.1 , then the null hypothesis was rejected, and non-inferiority was concluded. A non-inferiority test with a $25 \%$ non-inferiority margin was used for statistical analysis of the histology lateral thermal damage data. Typical thermal spreads for these devices were approximately $2.0 \mathrm{~mm}$, so $25 \%$ represents less than or equal to a $0.5 \mathrm{~mm}$ difference.

\section{Results}

\subsection{Bench top Testing}

\subsubsection{Lateral Thermal Spread}

IR camera imaging was used to capture and measure thermal spread on excised porcine carotid arteries at the moment of transection with HARMONIC 1100 and HD 1000i Shears. Table 1 lists the mean thermally affected zones (TAZ) measured along the arteries on either side of the device jaws. Sixteen examples of each device were fired twice in the analysis, for a maximum of 32 data points per device type. The mean TAZ measurement indicated no statistical difference in thermal spread between HARMONIC 1100 and HD 1000i Shears.

Table 1. Thermally affected zone (TAZ) of excised porcine carotid arteries transected with HARMONIC 1100 Shears (HAR1120 and HAR1136) compared to HARMONIC HD 1000i Shears (HARHD20 and HARHD36). *p-values for paired t-test of mean difference; (SD) standard deviation; $(N)$ number of transections.

\begin{tabular}{llll}
\hline Devices & N & $\begin{array}{l}\text { TAZ, mm, } \\
\text { mean (SD) }\end{array}$ & *P-Value \\
\hline HAR1120 & 29 & $3.61(0.83)$ & - \\
HARHD20 & 29 & $3.79(1.15)$ & - \\
Difference (HAR1120 - HARHD20) & 29 & -0.18 & 0.76 \\
HAR1136 & 32 & $3.50(0.79)$ & - \\
HARHD36 & 32 & $3.59(1.27)$ & \\
Difference (HAR1136 - HARHD36) & 32 & -0.09 & 0.62 \\
\hline
\end{tabular}

\subsubsection{Maximum Transient Blade Temperature}

Figure 2 depicts a generalized activation of the next generation Adaptive Tissue Technology in HARMONIC 1100 Shears during a single transection. When the predefined blade frequency / temperature threshold is reached, energy 
delivery to the HARMONIC 1100 is reduced to limit the maximum blade temperature and mitigate the potential for thermal damage. As indicated in Figure 2, tissue effects (sealing and cutting) are achieved at temperatures below the new algorithm threshold. To evaluate blade temperatures in benchtop studies, each device was used for three sets of 15 consecutive tip bites, and the overall mean maximum transient temperatures were compared between device types.
Table 2 lists the overall mean difference and percent increase in maximum temperatures for current Harmonic shears (HD 1000i and ACE + 7) compared to HARMONIC 1100 Shears (HAR1136), averaged across all 15 transections. A significantly lower maximum blade temperature was observed for HAR1136 relative to the other Harmonic devices $(\mathrm{p}<0.001$ for each comparison).

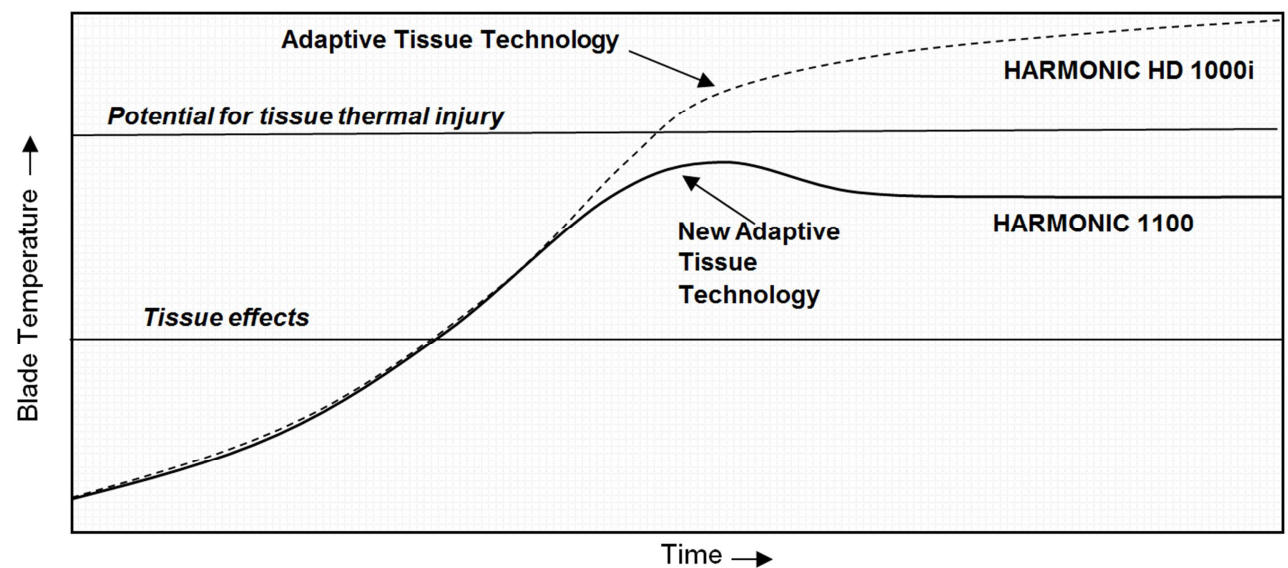

Figure 2. Activation of New Adaptive Tissue Technology in HARMONIC 1100 Shears during a single transection, in comparison with HD 1000i Shears.

Table 2. Percent increase and difference in overall mean transient maximum blade temperatures for Harmonic devices ( HD 1000i and ACE +7 ) compared to HARMONIC 1100. *p-values for mean differences.

\begin{tabular}{llll}
\hline Device & Max Temp relative to Harmonic 1100 (\% increase) & Mean difference from Harmonic 1100 & P-Value* \\
\hline HD 1000i & $+17.4 \%$ & $+49.1^{\circ} \mathrm{C}$ & $+56.8^{\circ} \mathrm{C}$ \\
$\mathrm{ACE}+7$ & $+20.1 \%$ & 0.001 & $<0.001$ \\
\hline
\end{tabular}

\subsubsection{Marching Transection Time}

The speed at which Harmonic shears completed 9 tip bite transections across porcine jejunum is shown in Table 3. A total of 32 devices of each type were used for determination of mean values for marching transection speed. No significant differences were observed between HARMONIC 1100 and HD 1000i, with devices completing the marching transection at mean times of 20.6 to 23.6 seconds. Full bite analysis also demonstrated no significant differences between devices (data not shown).

Table 3. Tip bite total marching transection time for HARMONIC 1100 Shears (HAR1120 and HAR1136) and HARMONIC HD 1000i Shears (HARHD20 and HARHD36), in bench-top analyses. (SD) standard deviation; Sample Size $=$ number of devices.

\begin{tabular}{lll}
\hline Device & Sample Size & Time, sec, mean (SD) \\
\hline HAR1120 & 32 & $20.6(1.32)$ \\
HARHD20 & 32 & $21.0(1.40)$ \\
HAR1136 & 32 & $23.3(1.72)$ \\
HARHD36 & 32 & $23.6(1.92)$ \\
\hline
\end{tabular}

\subsubsection{Burst Pressures}

The results of burst pressure testing for porcine carotid arteries transected and sealed with HARMONIC 1100 (HAR1136) and HD 1000i (HARHD36) are shown in Table 4. Five of each device type were used in the analysis. Mean burst pressures determined for vessels transected with the distal, middle, and proximal portion of device jaws did not show a statistical difference between HAR1136 and the predicate shears. These results demonstrated similar sealing strength for arteries of 3-5 mm diameter using the energy button for HARMONIC 1100 and HD 1000i.

Table 4. Burst pressures comparison of excised porcine carotid arteries sealed with HARMONIC 1100 Shears (HAR1136) and HARMONIC HD 1000i Shears (HARHD36). (SD) standard deviation; ${ }^{1}$ Total number of transections; ${ }^{2} p$-values for paired t-test comparisons.

\begin{tabular}{llll}
\hline Device/Jaw location & $\begin{array}{l}\text { Sample } \\
\text { Number }^{\mathbf{1}}\end{array}$ & $\begin{array}{l}\text { Burst Pressure, } \\
\text { mmHg, mean (SD) }\end{array}$ & P-Value $^{2}$ \\
\hline HAR1136/ Distal & 25 & $1192(578)$ & \\
HARHD36/ Distal & 25 & $1173(422)$ & 0.906 \\
Difference & 25 & 19 & \\
HAR1136/ Middle & 15 & $1291(397)$ & 0.176 \\
HARHD36/ Middle & 15 & $1469(190)$ & \\
Difference & 15 & -178 & 0.061 \\
HAR1136/ Proximal & 11 & $1438(260)$ & \\
HARHD36/ Proximal & 11 & $1109(402)$ & \\
Difference & 11 & 329 & \\
\hline
\end{tabular}

\subsection{In Vivo Acute Evaluation}

The proportion of hemostatic seals was extremely high (99.4\% for HARMONIC 1100 and $99.3 \%$ for HARMONIC HD 1000i Shears). Non-inferiority of HARMONIC 1100 compared to HD 1000i was demonstrated for both initial hemostasis and hemostasis at hypertensive challenge in either 
the Energy Activation mode or Advanced Hemostasis mode (Table 5). Non-inferiority was also shown for thermal damage of carotid arteries between the devices in both Energy Activation and Advanced Hemostasis modes (Table 5). Subjective evaluations of dissection capabilities were equally favorable for both HARMONIC 1100 and HD 1000i Shears. For the analysis of thoracic cavity applications, HARMONIC 1100 achieved hemostasis at 60 of 60 total sites (100\%), including 30 Energy Activation applications and 30 Advanced Hemostasis applications (data not shown). Similarly, HD 1000i achieved hemostasis at 60 of 60 overall sites $(100 \%)$.

Table 5. Hemostasis and lateral thermal damage in a porcine acute surgery model. *Mean (mm) \pm standard deviation.

\begin{tabular}{lll}
\hline Mode/Measure & $\begin{array}{l}\text { HARMONIC 1100 } \\
\text { (20 and 36cm) }\end{array}$ & $\begin{array}{l}\text { HARMONIC HD } \\
\mathbf{1 0 0 0 i}(\mathbf{2 0} \text { and 36 cm) }\end{array}$ \\
\hline Energy Activation Mode $(\leq 5 \mathrm{~mm}$ vessel diameter) & \\
Initial Hemostasis & $200 / 201(99.5 \%)$ & $183 / 183(100 \%)$ \\
Hemostasis at Challenge & $198 / 200(99.0 \%)$ & $181 / 183(98.9 \%)$ \\
Lateral Thermal Damage* & $2.15 \pm 0.45 \mathrm{~mm}$ & $2.0 \pm 0.55 \mathrm{~mm}$ \\
Advanced Hemostasis Mode $(\leq 7 \mathrm{~mm}$ vessel diameter $)$ \\
Initial Hemostasis & $153 / 154(99.4 \%)$ & $156 / 157(99.3 \%)$ \\
Hemostasis at Challenge & $152 / 153(99.3 \%)$ & $155 / 156(99.4 \%)$ \\
Lateral Thermal Damage* & $2.15 \pm 0.35 \mathrm{~mm}$ & $1.9 \pm 0.5 \mathrm{~mm}$ \\
\hline
\end{tabular}

\section{Discussion}

Ultrasonic surgical shears are advanced energy devices that use mechanical energy to seal vessels and transect tissue rather than the energy traveling to or through the patient. Mechanical compression, temperature / protein denaturation, and time are necessary to seal the vessels. Transection can be accomplished by simply activating the device. Advantages of using ultrasonic devices are multifactorial: the desired tissue effect can be regulated by the generator delivering the mechanical energy, surrounding tissues have reduced thermal damage, the energy is designed to keep heat in the blade rather than passing it through patient tissues, there is minimal lateral thermal spread, simultaneous cutting and coagulation, and reduced plume.

The primary objective of this study was to evaluate performance of the next generation Harmonic device (HARMONIC 1100 Shears) in the context of a newly designed Adaptive Tissue Technology energy delivery system. It was determined that the maximum blade temperatures using HARMONIC 1100 in marching transections were significantly lower compared to the previous HD 1000i device. The Adaptive Tissue Technology function is activated only in the Energy Activation mode of the device and does not affect activations with the Advanced Hemostasis button, which employs a separate monitoring algorithm. As the HARMONIC 1100 is activated, software on the generator monitors changes in blade characteristics such as frequency and impedance. The new Adaptive Tissue Technology algorithm reduces energy delivery to the device once the blade reaches predefined frequency and temperature values. The system is designed to provide effective transection and sealing during surgical procedures while mitigating the risk of thermal tissue injury or possible premature tissue pad wear on the device jaws when used under conditions that would generate the most heat. Bench top testing including lateral thermal spread and transection speed confirmed similar results for HARMONIC 1100 and HD 1000i Shears. In addition, the carotid artery burst pressures demonstrated no loss of transection capability and sealing quality for the new device under the Energy mode, indicating no negative consequence on vessel transection with the enhanced Adaptive Tissue Technology system.

The in vivo porcine studies demonstrated successful hemostasis upon sealing and transection of a variety of major vessels with the HARMONIC 1100 Shears. Ninety-nine percent of the vessels tested achieved hemostasis with initial activation of the new Harmonic device, which was not statistically different than hemostasis rates found in comparison with HD 1000i. This high rate of first pass hemostasis was also observed when the pigs were subjected to a hypertensive challenge. Thermal tissue damage, assessed by histological analysis of the carotid artery seal, was limited to a mean of $2.15 \mathrm{~mm}$, which met the requirement of noninferiority compared to HD 1000i. The analyses also included activation of the Advanced Hemostasis mode for transecting vessels up to and including $7 \mathrm{~mm}$ in diameter. Both the new 1100 and HD $1000 \mathrm{i}$ devices achieved successful hemostasis in more than $99 \%$ of vessels in both energy modes. These data show effective, non-inferior hemostatic sealing of vessels in vivo with the HARMONIC 1100 Shears, with no adverse effect of the new Adaptive Tissue Technology algorithm.

\section{Conclusion}

The next generation of Adaptive Tissue Technology is embodied in HARMONIC 1100 Shears. This latest advancement maintains all the previous benefits of HARMONIC HD 1000i at lower maximum blade temperatures. Multiple clinical studies have shown the effective use of the current HD 1000i Shears in various procedures including pancreatectomy [12], breast reconstruction capsulectomy [13], laparoscopic hysterectomy [14], and esophagectomy [15]. Benchtop and preclinical results in this study suggest similar performance for the HARMONIC 1100 Shears, and it is anticipated that clinical results will also be comparable.

\section{References}

[1] Devassy R, Gopalakrishnan S, De Wilde RL. Surgical Efficacy Among Laparoscopic Ultrasonic Dissectors: Are We Advancing Safely? A Review of Literature. J Obstet Gynaecol India 2015; 65: 293-300.

[2] Law KS, Abbott JA, Lyons SD. Energy sources for gynecologic laparoscopic surgery: a review of the literature. Obstet Gynecol Surv 2014; 69: 763-76. 
[3] Lyons SD, Law KS. Laparoscopic vessel sealing technologies. J Minim Invasive Gynecol 2013; 20: 301-7.

[4] Wang K, Advincula AP. "Current thoughts" in electrosurgery. International journal of gynaecology and obstetrics: the official organ of the International Federation of Gynaecology and Obstetrics 2007; 97: 245-50.

[5] Cannizzaro MA, Borzi L, Lo Bianco S, Okatyeva V, Cavallaro A, Buffone A. Comparison between Focus Harmonic scalpel and other hemostatic techniques in open thyroidectomy: A systematic review and meta-analysis. Head and Neck 2016; 38 (10): 1571-8.

[6] Cheng H, Hsiao CW, Clymer JW, et al. Gastrectomy and D2 Lymphadenectomy for Gastric Cancer: A Meta-Analysis Comparing the Harmonic Scalpel to Conventional Techniques. Int J Surg Oncol 2015; 2015: 397260.

[7] Ren ZH, Xu JL, Fan TF, Ji T, Wu HJ, Zhang CP. The Harmonic Scalpel versus Conventional Hemostasis for Neck Dissection: A Meta-Analysis of the Randomized Controlled Trials. PLoS One 2015; 10: e0132476.

[8] Revelli L, Damiani G, Bianchi CB, et al. Complications in thyroid surgery. Harmonic Scalpel, Harmonic Focus versus Conventional Hemostasis: A meta-analysis. International journal of surgery 2016; 28 Suppl 1: S22-32.

[9] Cheng H, Clymer JW, Ferko NC, et al. A systematic review and meta-analysis of Harmonic technology compared with conventional techniques in mastectomy and breast-conserving surgery with lymphadenectomy for breast cancer. Breast Cancer (Dove Med Press) 2016; 8: 125-40.
[10] Cheng H, Soleas IM, Ferko NC, Cameron CG, Clymer JW, Amaral JF. Hospital costs associated with thyroidectomy performed with a Harmonic device compared to conventional techniques: a systematic review and meta-analysis. Journal of Medical Economics 2016; 19 (8): 750-8.

[11] Zhang ZJ, Zhang P, Tian JH, et al. Ultrasonic coagulator for thyroidectomy: a systematic review of randomized controlled trials. Surgical innovation 2010; 17: 41-7.

[12] Casadei R, Ricci C, Pacilio CA, Ingaldi C, Taffurelli G, Minni F. Laparoscopic distal pancreatectomy: which factors are related to open conversion? Lessons learned from 68 consecutive procedures in a high-volume pancreatic center. Surg Endosc 2018; 32: 3839-45.

[13] Kim KJ, Chung JH, Lee HC, Lee BI, Park SH, Yoon ES. Comparison of Harmonic scalpel and monopolar cautery for capsulectomy at the second stage of expander/implant breast reconstruction. Arch Plast Surg 2020; 47: 140-5.

[14] McCarus SD. McCarus Minimally Invasive Hysterectomy: 20 Years' Experience-Lessons Learned. Surgical technology international 2018; 33: 207-14.

[15] Otsuka K, Murakami M, Goto S, et al. Minimally invasive esophagectomy and radical lymph node dissection without recurrent laryngeal nerve paralysis. Surg Endosc 2020; 34: 2749-57. 